\title{
PPU for a small, low power and low cost EPS
}

\author{
Spiridon Savvas ${ }^{1, *}$, Pavlos Ramnalis ${ }^{1}$, and Alexandros M anoudis $^{1}$ \\ ${ }^{1}$ SITAEL Hellas, Dionisiou Solomou 4, 59132 V eria, Greece
}

\begin{abstract}
The objective of this paper is to present the design of the Power Processing Unit (PPU) of the M icrosatellite Electric Propulsion System (MEPS) program currently being developed at Elegant B read Board (EBB). High efficiency, small size and weight and high reliability are the main parameters that special focus is given. The propulsion system of MEPS consists of two small size and low power (up to 300W) Thruster Units (TUs) each one composed of a Hall Effect Thruster and a Cathode, a single Propellant $M$ anagement and Tank A ssembly (PM A/PTA) and a single PPU. The PPU designed for the EBB phase has no redundancy, is fully flexible and capable of driving two different TU types (Rafael's CAM 200 coupled with Rafael's Heaterless Hollow Cathode (RHHC) and SITAEL's HT100 coupled with SITAEL's Heated Hollow Cathode ( $\mathrm{HC} 1)$ ). This paper illustrates the core design requirements and important aspects on which the design was based. Preliminary results obtained from the coupling tests on the critical parts developed at Bread Board (BB), which contributed to the current design are also briefly depicted. Last but not least, the future development steps of the program that can become the driving factor for the successful implementation of an easily adjustable PPU compatible with a wide range of low power Electric Propulsion Systems (EPSs) are demonstrated.
\end{abstract}

\section{Introduction}

MEPS program has been originated by the increasing need to provide a low-cost and low-power Electric Propulsion System (EPS) for small satellites $(<300 \mathrm{Kg})$ and finds applications in orbit insertion after launcher separation, orbit maintenance, orbit transfers and deorbiting.

The EPS of MEPS consists of two TUs, each one composed of a Hall Effect Thruster and a Cathode, a single PMA/PTA and a single PPU.

The PPU constitutes one of the most challenging parts, from both technical and business point of view, of an EPS. It is the module that contains the electrical circuits responsible for providing electrical power and control to all the other subsystems with varying demands and in the most efficient way, while its cost is indicatively about $50 \%$ of the total cost of an EPS. Space industries are strongly focusing on the development of low cost PPUs trying to introduce small in size and weight units with high efficiency and reliability.

PPU, as conceived for MEPS program, is supplied by the satellite's Power Distribution Unit (PDU) and has the following main functions:

- Power conditioning and monitoring of all voltages and currents required by the EPS in all of its operating modes.

- To enable the control of the EPS in order to achieve the best performance according to mission requirements.

- To manage bidirectional communication with satellite computer including Telemetries (TMs) and Telecommands (TCs).

\footnotetext{
${ }^{*}$ Corresponding author: spiridon.savvas@ sitael-hellas.com
}

- To ensure isolation between the high voltage electronics that supply the thrusters and the low voltage electronics supplied by the input power bus.

- To protect the main bus from the TU EMI.

In the framework of MEPS program, SITAEL Hellas, supported by SITAEL, is responsible for designing the PPU of the EPS. The PPU is currently being developed in TRL 4, Elegant Breadboard (EBB) and the target is in 2 years frame to reach TRL 6. Its electrical design has been recently approved by ESA and will be manufactured and tested during the next phases.

Complying with the latest system specifications, the PPU EBB is able to drive two different thruster units utilizing the same PMA/PTA (Fig. 1):

- Rafael's TU composed of CAM-200 Thruster coupled with RHHC Cathode (R-TU).

- SITAEL's TU composed of HT100 Thruster coupled with $\mathrm{HC} 1$ cathode (S-TU).

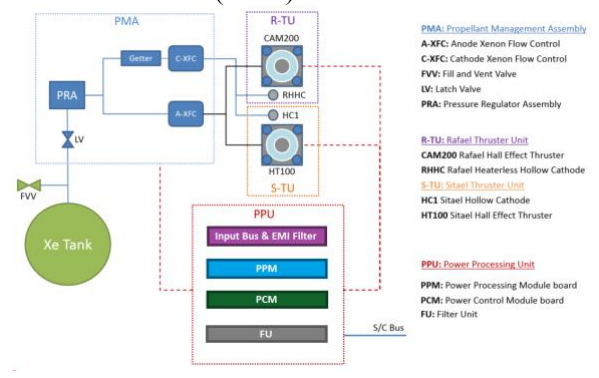

Fig. 1. MEPS Architecture 
Fig. 1 presents the general architecture of MEPS. The PPU developed in the EBB phase is non-redundant and contains dedicated modules able to drive either R-TU or S-TU system.

\section{Power Processing Unit}

The PPU enables the single operation of any one of the thruster units in their complete thrust range, ensures full performance for an unregulated input power bus voltage from $22 \mathrm{~V}$ to $34 \mathrm{~V}$, it does not suffer any damage up to $40 \mathrm{~V}$ input bus voltage and is composed of the following functional blocks:

- Input Power Bus Switch and Input EMI filter which implement the ON/OFF of the PPU Power Module providing protection and stability of the whole system.

- PPU Power Module (PPM) which implements the power electronic circuits necessary to supply any of the two Thruster Units.

- PPU Control Module (PCM), which provides the communication with the satellite and the control of all the PPU modules related with the TUs and the PMA/PTA.

- Filter Unit (FU) which has the purpose to filter the high frequency current noise typically associated with the TUs.

Although the PPU is currently being developed in an EBB level with commercial equivalent components, the design was optimized and the relevant analyses have been implemented with Flight Model (FM) components in order to identify possible issues and foresee any required modifications for the later development phases. The design is compatible with a total ionizing dose of $30 \mathrm{Krad}$ and the selected components are free from destructive single event effects (SEL, SEB, SEGR) up to $60 \mathrm{MeV}$, which is the threshold generally considered to define a part free from SEE. The top level design requirements of the PPU are:

- Reliability of at least 0.99 .

- Efficiency higher than $92 \%$ at maximum input power of $300 \mathrm{~W}$ during steady state operation.

- Absorbed electric power from input bus less than 10W at standby state.

- Mass lower than $7 \mathrm{~kg}$.

- The envelope of PPU size shall be less than $300 \mathrm{~mm} \mathrm{x}$ $240 \mathrm{~mm} \times 230 \mathrm{~mm}$.

The different PPU modules are thoroughly described in the following sections.

\subsection{Input Power Bus Switch and Input EMI filter}

The Input Power Bus Switch is able to disconnect the PPM from the bus in case of internal failures causing an unexpected absorbed current above a predefined threshold as it is equipped with a high-speed overcurrent latching protection. Consequences of a short to structure failure, not covered by this module, are mitigated since an external LCL is foreseen at system level. Furthermore, it allows the PPU switching ON and OFF by TC provided by the PCM and provides in-rush current control.
The Input EMI filter attenuates the noise to/from input power bus in order to be compliant with EMC requirements and guarantees the stability of overall system.

\subsection{PPM}

PPM is the module that includes the DC/DC power supplies necessary to supply the TUs of both R-TU and S-TU and the PMA/PTA. Specifically it implements:

- The Anode Power Supply (APS) for both systems.

- The Cathode Power Supply (CAPS) for both systems.

- The Cathode Power Supply High Voltage (CAPS HV) for R-TU.

- The Magnetic Field Power Supply (MGFPS) for R-TU.

- The Cathode Heater Power Supply (CHPS) for S-TU.

- The Auxiliary Power Supply (AUXPS) for all housekeeping subsystems and PMA/PTA driving.

\subsubsection{APS}

APS constitutes the most critical PPU module since it manages the highest power demands supplying the Thrusters of the EPS. It supports the plasma discharge current and sets the anode voltage during operation for HT100 and CAM-200 Thrusters. APS design approach is common for R-TU and S-TU, as an envelope of the requirements for CAM-200 and HT100 has been considered.

APS is implemented via an isolated DC/DC power supply whose output is connected between the thruster anode and the Cathode Reference Potential (CRP). It is supplied by the primary input power Bus downstream the Input Power Bus Switch and Input EMI filter. It provides:

- Power up to $250 \mathrm{~W}$ to drive the thruster anode during steady state operation.

- Acquisition of anode temperature, driving voltage and discharge current.

- Regulation of anode voltage according to the thrust required by the satellite $\mathrm{OBC}$

- Galvanic isolation between input power bus and the thrusters. The isolation voltage is $1000 \mathrm{Vdc}$.

The electrical characteristics of APS are summarized in Table 1

Table 1. APS electrical characteristics

\begin{tabular}{|c|c|c|}
\hline APS & $\min$ & $\mathbf{m a x}$ \\
\hline Output Voltage range [V] & 200 & 400 \\
\hline Output Current range [A] & 0.4 & 1.1 \\
\hline Output Power range [W] & 100 & 250 \\
\hline
\end{tabular}

The implemented DC/DC converter is based on an asymmetrically controlled half bridge converter with a boost conversion in the front stage and a voltage doubler rectifier in the output stage (derived from the BoostIntegrated Half Bridge (BHB) topology). Therefore, a 
magnetic component is avoided, yielding the reduction of weight and power losses, and the transformer realization is simpler because the voltage doubler does not need the central tap of the transformer secondary windings. High voltage isolation has been considered within the output voltage feedback circuit, the voltage and current monitors and the overvoltage protection circuit. Average current mode control technique is used for better stability and more precise control of the input current. In addition, outer loop has been used to control also the output voltage. Last but not least, the modified BHB topology allows both the Zero Voltage Switching transitions of the primary MOSFETs and the Zero Current Switching transitions of the secondary diodes, permitting a high-efficiency even with a relative low power.

The APS converter has been initially validated using a dynamic load simulator with an output operating voltage of $300 \mathrm{~V}$ and currents between $0.3 \mathrm{~A}$ and $1 \mathrm{~A}$, which are encountered with the various flow rates. Fig. 2 presents the test results at three input voltages $(22 \mathrm{~V}, 28 \mathrm{~V}$ and $34 \mathrm{~V}$ ) with dummy resistive loads.

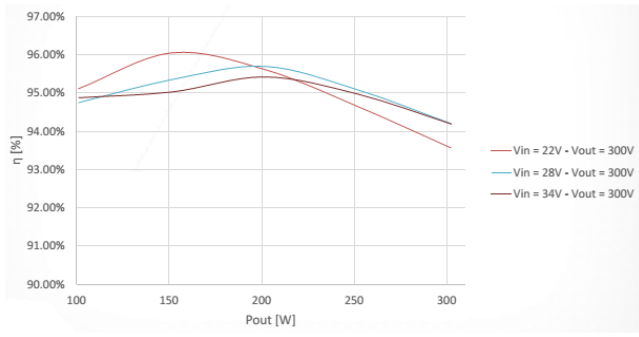

Fig. 2. Efficiency for output Voltage $300 \mathrm{~V}$ and different Input Voltages

\subsubsection{CAPS}

CAPS is an isolated DC/DC Power Supply, based on a standard flyback topology providing the following features:

- High voltage driving of HC1 Cathode.

- Current driving during keeping phase.

- Acquisition of cathode driving voltage and current

- Galvanic isolation between input power bus and the thruster and the chassis.

CAPS is supplied, by the power bus via the input power interface. Thus, it is electrically placed downstream the bus switch and the input EMI filter. Its main electrical characteristics are depicted in Table 2.

Table 2. CAPS electrical characteristics

\begin{tabular}{|l|c|c|}
\hline \multicolumn{1}{|c|}{ CAPS } & $\min$ & $\max$ \\
\hline Output Voltage [V] & - & 500 \\
\hline Output Current range [A] & 0.05 & 1 \\
\hline Output Power [W] & - & $\begin{array}{c}30(\mathrm{HC} 1) \\
40(\mathrm{RHHC})\end{array}$ \\
\hline
\end{tabular}

CAPS design approach is common for both R-TU and S-TU system. The main differences between the module that is able to drive RHHC and the one that is able to drive $\mathrm{HC1}$, are related to the maximum output power (40W for RHHC and $30 \mathrm{~W}$ for $\mathrm{HC} 1$ ) and to the current keeping steps (11 steps for RHHC and 8 steps for $\mathrm{HC} 1)$.

\subsubsection{CAPS HV Igniter}

An isolated HV module (set at $800 \mathrm{~V}$ ) has been implemented as a breakdown initiator by charging dedicated capacitors that will provide the required energy to the cathode supporting the ignition sequence of the RHHC and so concerns only the R-TU system.

It will operate for a short time only during RHHC ignition process and it will initiate before CAPS main module operation that will then support the keeping phase after the breakdown.

Since this module will be ON only for a short time, a simple and flexible DC/DC converter (a flyback topology with two Cockcroft-Walton (CW) voltage multipliers in the output) has been designed.

HV module is supplied, as APS and MGFPS, by the power bus via the input power interface. So it is electrically placed downstream the bus switch and the input EMI filter.

\subsubsection{MGFPS}

MGFPS is an isolated DC/DC settable current source whose purpose is to supply the magnetic field coils of the CAM-200 thruster with a current up to 3A. This module concerns only the R-TU system since the S-TU system and specifically the HT100 thruster has permanent magnets.

The MGFPS module is connected to the unregulated bus coming from the PDU downstream the EMI input filter and inrush current control circuit.

In order to improve the efficiency and reliability of the PPU, the MGFPS is implemented with only one isolated conversion stage, with forward topology integrating voltage and power limitation circuits. MGFPS efficiency for a typical operating point of Iout $=2 \mathrm{~A}$ and Pout $=7.2 \mathrm{~W}$ is calculated at $\sim 85 \%$.

The electrical characteristics of MGFPS are illustrated in Table 3.

Table 3. MGFPS electrical characteristics

\begin{tabular}{|l|c|c|}
\hline \multicolumn{1}{|c|}{ MGFPS } & min & max \\
\hline Zout (coils impedance) & \multicolumn{2}{|c|}{$1.5 \mathrm{mH}+1.8 \Omega$} \\
\hline Output Voltage range [V] & 0 & 12 \\
\hline Output Current range [A] & 0.1 & 3 \\
\hline Output Power range [W] & 0.1 & 23 \\
\hline
\end{tabular}




\subsubsection{CHPS}

CHPS is a non-isolated DC/DC settable current source related only to the driving of S-TU system. Specifically, its purpose is to supply the Cathode Heater of SITAEL's $\mathrm{HC} 1$ heated cathode with a current up to 7A.

CHPS main electrical characteristics are summarized in Table 4.

Table 4. CHPS electrical characteristics

\begin{tabular}{|c|c|c|}
\hline CHPS & min & max \\
\hline Output Voltage range [V] & 0 & 12 \\
\hline Output Current range [A] & 0 & 7 \\
\hline Output Power range [W] & 0 & 50 \\
\hline
\end{tabular}

The CHPS module is connected to the unregulated bus coming from the PDU downstream the EMI input filter and inrush current control circuit.

For the CHPS a synchronous buck converter is utilized, since no isolation is required (the required isolation is achieved due to the hardware implementation of the heater inside the cathode).

\subsubsection{AUXPS}

AUXPS consists of the following parts that are thoroughly described in this chapter:

- AUXPS Main DC/DC converter.

- PMA/PTA distribution and valves driving.

- APS/CAPS secondary auxiliary supply (Flyback Converter).

AUXPS Main DC/DC converter is directly supplied by the input power bus. An EMI filter connected downstream the input power bus allows to reduce electromagnetic conducted emission toward input bus lines and increase output voltage immunity in presence of input voltage transient variations.

The AUXPS Main is implemented via a multiple output Forward DC/DC converter with peak current mode control operating in continuous conduction mode. The nominal switching frequency is around $100 \mathrm{kHz}$ in free-running mode and the efficiency has been calculated at $85 \%$ for typical AUXPS operation.

Moreover, the galvanically isolated Auxiliary Main DC/DC converter provides all the housekeeping supplies necessary to the PPU modules and subcircuits and input power supplies for the other two parts:

PMA/PTA part is supplied with the required steady state and activation voltages. It consists of the following parts: 2 High pressure transducers (HPT), 2 Low pressure transducers (LPT), 2 Heaters (HTR), 6 DXFC valves (DXFCV), 4 Isolation valves for each TU (IV) and 2 Latch valves (LV).

Finally, a dedicated flyback DC/DC converter is implemented in order to supply the HV secondary side of APS and CAPS with the required auxiliary power supplies. Galvanic isolation between primary and secondary (HV) side is achieved via a transformer with double isolation. The flyback DC/DC converter implements three auxiliary rectified outputs.

\subsection{PCM}

The EPS is externally controlled by the spacecraft's OnBoard Computer (OBC) while its internal control is performed by the PCM.

In particular the PCM controls the APS, CAPS, CAPS HV Igniter, AUXPS and MGFPS or CHPS and the PMA/PTA valves so as to enable the driving and control of one thruster at a time.

Specifically, the PCM provides the following features:

- Temperature monitor via thermal sensors located in the PPU, Thrusters and PMA.

- Anode and cathode driving voltage and current monitors.

- Magnetic field voltage and current monitor in case of Rafael's TU.

- Cathode heater voltage and current monitor in case of SITAEL's TU.

- PMA Heaters and valves driving voltage monitors.

- Voltage settings for APS and current settings for CAPS keeping phase and MGFPS (for R-TU) or CHPS (for S-TU).

- Sequencing and management of thruster's operation in various modes (i.e. start-up, shutdown, power setting, thrust regulation and failure recovery).

- TM/TC management.

The PCM is an FPGA-based digital module including non-volatile memory with EDAC for parameters storage, ADCs and MUXes (with relative signals conditioning circuits) for external/internal signals readouts and sets for the Anode voltage and Cathode and Magnet (or Cathode Heater) currents, as well as all the required commands.

The FPGA approach will make the programmed sequences control more flexible with the possibility of adjusting the internal parameters.

In the current phase of the program, two PCM modules have been designed; one for the R-TU system and one for the S-TU system.

\subsection{FU}

FU is a passive low pass LC filter with resistive damping which is aimed at reducing the high frequency noise current generated by the TU Anode during its operation. A dedicated capacitor has been implemented in CAPS output in order to deliver the appropriate energy to the keeper during the breakdown event.

The capability of FU will be evaluated during the coupling test activities and its operation shall be optimized in order to be compatible with the Electromagnetic compatibility (EMC) and Electrostatic discharge (ESD) requirements.

In the current phase of the program, two FUs are utilized; one for the R-TU system and one for the S-TU system. 


\section{Coupling Tests}

In the framework of MEPS project, a PPU BB has been developed with the most critical parts (APS and CAPS) and coupling tests have been performed in order to verify its capability to ignite the RHHC with both HETs (in the initial concept and at that time being, RHHC was the only declared Cathode in the program capable of working with both HETs). Fig. 5 illustrates a capture of HT100 coupled with RHHC firing during the coupling tests.

The current PPU EBB design of the critical parts (particularly APS) is based also on the results and the lessons learnt from these tests. Regarding the less critical parts of the PPU EBB, that will be developed for the first time at this phase, their functionality will be verified during the foreseen EBB tests.

\subsection{PPU-CAM-200 Coupling Tests}

The coupling tests with the PPU BB succeeded the ignition of CAM-200 thruster in the five operating points recorded in Table 5. A node and Cathode Mass Flow Rates ( $A_{M F R}$ and $C_{M F R}$ respectively) together with APS output Voltage and Power (V OUt_AN and POUt_AN respectively) are also presented in Table 5.

Table 5. CAM-200: Operating points

\begin{tabular}{|c|c|c|c|c|}
\hline $\begin{array}{c}\text { Operating } \\
\text { Point }\end{array}$ & $\begin{array}{c}\text { AMFr } \\
{[\mathbf{m g} / \mathbf{s}]}\end{array}$ & $\begin{array}{c}\text { CMFR } \\
{[\mathbf{m g} / \mathbf{s}]}\end{array}$ & $\begin{array}{c}\text { Vout_AN } \\
{[\mathbf{V}]}\end{array}$ & $\begin{array}{c}\text { Pout_AN } \\
{[\mathbf{W}]}\end{array}$ \\
\hline 1 & 0.62 & 0.25 & 250 & 110 \\
\hline 2 & 0.69 & 0.25 & 250 & 130 \\
\hline 3 & 0.75 & 0.25 & 275 & 160 \\
\hline 4 & 0.83 & 0.25 & 275 & 180 \\
\hline 5 & 0.9 & 0.25 & 275 & 200 \\
\hline
\end{tabular}

During the coupling tests, APS efficiency was measured in each operating point for three different input voltages $(22 \mathrm{~V}, 28 \mathrm{~V}$ and $34 \mathrm{~V})$. The related results are presented in Fig. 3.

As shown in Fig. 3, APS efficiency exceeded 92\% for the five operating points listed in Table 5 . The results deemed to be promising in order to achieve the final target of exceeding the $92 \%$ overall efficiency for the PPU in the next phases of the program.

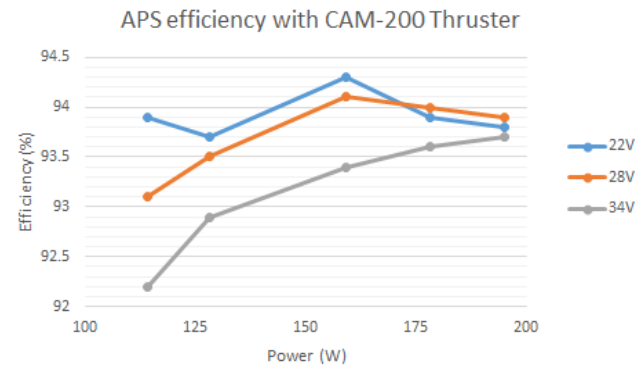

Fig. 3. APS efficiency of CAM-200-different input voltages

\subsection{PPU-HT100 Coupling Tests}

The coupling tests with the PPU BB succeeded the ignition of HT100 thruster in the five operating points recorded in Table 6 . A node and Cathode $M$ ass Flow Rates ( $A_{M F R}$ and $C_{M F R}$ respectively) together with APS output Voltage and Power (V OUT_AN and POUT_AN respectively) are also presented in Table 6 .

Table 6. HT100: Operating points

\begin{tabular}{|c|c|c|c|c|}
\hline $\begin{array}{c}\text { Operating } \\
\text { Point }\end{array}$ & $\begin{array}{c}\text { AMFR } \\
{[\mathbf{m g} / \mathbf{s}]}\end{array}$ & $\begin{array}{c}\text { CMFr } \\
{[\mathbf{m g} / \mathbf{s}]}\end{array}$ & $\begin{array}{c}\text { Vout_AN } \\
{[\mathbf{V}]}\end{array}$ & $\begin{array}{c}\text { PouT_AN } \\
{[\mathbf{W}]}\end{array}$ \\
\hline 1 & 0.6 & 0.25 & 300 & 130 \\
\hline 2 & 0.75 & 0.25 & 275 & 160 \\
\hline 3 & 0.75 & 0.25 & 325 & 190 \\
\hline 4 & 1 & 0.25 & 275 & 220 \\
\hline 5 & 1 & 0.25 & 300 & 250 \\
\hline
\end{tabular}

During the coupling tests, APS efficiency was measured in each operating point for three different input voltages $(22 \mathrm{~V}, 28 \mathrm{~V}$ and $34 \mathrm{~V})$. The related results are presented in Fig. 4.

As shown in Fig. 4, APS efficiency exceeded 93\% for the five operating points listed in Table 6 . In that case also the results are promising in order to achieve the final target of exceeding the $92 \%$ overall efficiency for the PPU in the next phases of the program.

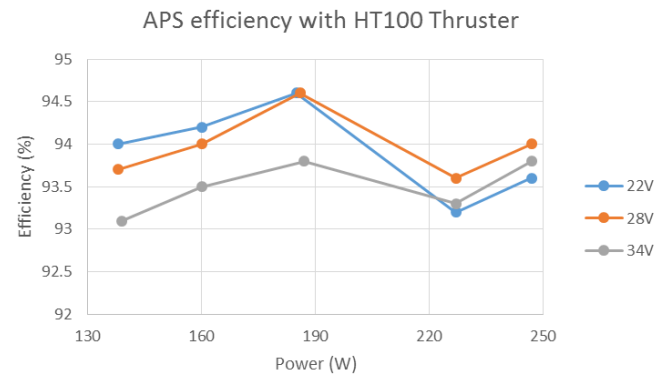

Fig. 4. APS efficiency of HT100-different input voltages

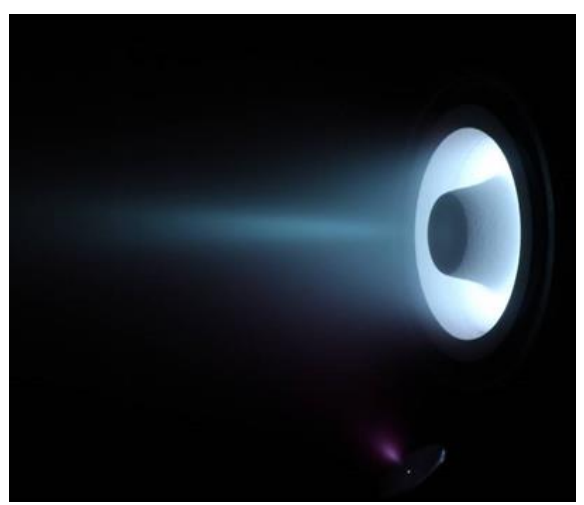

Fig. 5. HT100 on fire 
PPU CAPS for RHHC has also been tested at BB level and complied with the requirements so far. However, during the development of MEPS program, CAPS requirements for RHHC have been modified significantly. Consequently, the design concept is new and the results of the coupling tests had minor impact on it. Moreover, the latest introduction of the $\mathrm{HCl}$ in the program led to the redefinition of the design concept; CAPS will be extensively tested along with the other PPU modules during the foreseen EBB testing activities.

\section{Budget Analyses}

The efficiency, mass, size, weight and reliability analysis of the PPU has been performed for the EBB phase and the results are recorded hereafter. Worth to be noted is that for the necessary calculations the FM components of the final product have been taken into account. Particularly, all the relevant analyses concern separately the two systems (R-TU and S-TU); from now on, for distinction reasons we will be referring to the PPU that is able to drive the R-TU system as PPU R-TU and the PPU that drives the S-TU as PPU S-TU.

PPU Power Budget of the steady state case of the firing thrusters, has been estimated considering nominal input bus and worst combination of maximum load conditions required by the HT100 and CAM-200 thrusters as came out by the coupling tests; specifically, the following values have been used:

- Nominal Input Voltage: 28V (R-TU and S-TU).

- APS Vout $=250 \mathrm{~V}$; Iout $=1 \mathrm{~A}(\mathrm{Po}=250 \mathrm{~W})(\mathrm{R}-\mathrm{TU}$ and $\mathrm{S}$ TU).

- CAPS and CAPS HV Igniter for RHHC: OFF (R-TU).

- CAPS for HC1: OFF (S-TU).

- MGFPS Output Current 2A (R-TU).

- CHPS: OFF (S-TU).

- 2 Isolation Valves ON (R-TU and S-TU).

Table 7 summarizes the results and presents the data for PPU R-TU and PPU S-TU. Although the efficiency for both units is slightly lower than the initial requirement, it is believed that with the foreseen optimizations the targeted value can be achieved in the following stages of the program.

Table 7. PPU power budget

\begin{tabular}{|c|c|c|c|c|}
\hline & Pout $_{\text {oW] }}$ & P $_{\text {in }}[\mathbf{W}]$ & P diss $_{\text {[W] }}$ & n [\%] \\
\hline PPU R-TU & 263.95 & 289.89 & 25.94 & 91.1 \\
\hline PPU S-TU & 256.65 & 281.18 & 24.53 & 91.3 \\
\hline Reqs & & $<300$ & $<24$ & $>92$ \\
\hline
\end{tabular}

Table 8 presents the estimation for the total number of the EEE components and their corresponding weight for both PPU R-TU and PPU S-TU. The fulfilment of the initial mass requirement (PPU to be lower than $7 \mathrm{~kg}$ ) will be evaluated at the next phases of the program since it concerns a redundant PPU including the mechanical frames.
Table 8. PPU EEE components number and mass budget

\begin{tabular}{|c|c|c|}
\hline & Components number & Mass [g] \\
\hline PPU R-TU & 3076 & 2050 \\
\hline PPU S-TU & 2814 & 1910 \\
\hline
\end{tabular}

Specific attention has been paid during the design on the area budget of the PPU. The calculated results are illustrated in Table 9.

Table 9. PPU EEE components area budget

\begin{tabular}{|c|c|}
\hline & Components occupation area $\left[\mathbf{m m}^{\mathbf{2}}\right]$ \\
\hline PPU R-TU & 183262 \\
\hline PPU S-TU & 172337 \\
\hline
\end{tabular}

Detailed reliability analysis has also been performed for each PPU subassembly during the PPU EBB development. The reliability budget for PPU R-TU and PPU S-TU is depicted in Table 10. Failure rates (FR) have been calculated at both $25^{\circ} \mathrm{C}$ and $50^{\circ} \mathrm{C}$ for each component.

Table 10. PPU reliability budget

\begin{tabular}{|c|c|c|}
\hline & FR $\left({ }^{*} \mathbf{1 0}^{-\mathbf{9}}\right) @ \mathbf{2 5}^{\circ} \mathbf{C}$ & FR $\left({ }^{*} \mathbf{1 0}^{-\mathbf{9}}\right) @ \mathbf{5 0}{ }^{\circ} \mathbf{C}$ \\
\hline PPU R-TU & 614 & 1067 \\
\hline PPU S-TU & 521 & 916 \\
\hline
\end{tabular}

Considering the budget analysis performed during the EBB phase, the results seem to be quite promising. Nevertheless, further optimizations are expected during the next stages of the project. The EBB coupling tests are expected to contribute significantly towards that direction.

\section{Future Steps}

Currently, the PCB design of the PPU subassemblies is ongoing in parallel with the procurement of the EEE components. Upon the completion of the PCB design, the mechanical design will be performed and the PPU EBB MRR, which will mark the beginning of the manufacturing phase is scheduled to be held in the next months. The plan thereafter, includes thorough standalone and coupling tests in order to verify its capability to drive both TUs (Rafael's CAM-200 coupled with RHHC and SITAEL's HT100 coupled with HC1) which share the same PMA/PTA developed by Rafael. The tests are scheduled for next year.

Upon the successful completion of the tests, where the system requirements will freeze, any related modifications will be applied and the PPU will be developed then at TRL 6, Engineering Qualification Model (EQM). 


\section{Conclusions}

In the framework of MEPS program, SITAEL Hellas, supported by SITAEL, is responsible for designing a PPU that will be able to drive a low power EPS. The under development PPU EBB will be able to drive two different thruster units utilizing the same PMA/PTA; SITAEL's TU composed of HT100 Thruster coupled with $\mathrm{HCl}$ cathode (S-TU) and Rafael's TU composed of CAM-200 Thruster coupled with RHHC Cathode (RTU).

The PPU EBB design is presented in this paper via the description of its discrete modules. The PPU requirements have been defined taking into account the specifications derived from both systems (R-TU and STU) and wherever possible, the same module has been used for driving the two systems.

The design of the PPU EBB was based on the critical testing, remarks and development of the power supplies at BB level that showed successful and repeatable results in different configurations. Indicative results from the coupling tests performed with RHHC and CAM-200 or HT100 Thrusters are recorded for the scope of this paper.

Moreover, a summarized budget analysis for the PPU EBB is presented in order to depict the current status of the development. The calculations and estimations illustrated have been performed taking into account the FM components in order to reflect the final product characteristics.

Even if the results seem to be really encouraging, further design optimizations are foreseen during the next stages of the project where the PPU EBB will be manufactured and thoroughly tested at stand-alone level and coupled with the other EPS subsystems.
In addition, MEPS PPU development creates a significant heritage in the challenging power electronics field of EPS in general and particularly in Greece. Last but not least, it is strongly believed that MEPS PPU development can become the driving point for the successful implementation of an easily adjustable PPU compatible with a wide range of low power Electric Propulsion Systems.

\section{References}

1. S. Savvas, D. Malamas, A. Manoudis, P. Ramnalis, L.B enetti, S. d'Argliano, L. Onida, D. Caniglia, L.Fontani, T. Misuri, "Power Processing Unit for Microsatellite Electric Propulsion System", ESPC 2016, E3S W eb of Conferences 16, 15004 (2017)

2. T. M isuri, C. Ducci, L. Benetti, M. A ndrenucci, B. Waldvogel, D. Lev, G. Alon, J. Herscovitz, K. Dannenmayer, "MEPS Engineering Model Development and Test" , Space Propulsion 2016 , Rome, Italy , 2-6 M ay 2016

3. T. Misuri, C. Ducci, R. Albertoni, M. Andrenucci, D. Pedrini, "Sitael Low Power Hall Effect Thrusters for Small Satellites", IEPC-2015-102 ISTS-2015-b102, Kobe, Japan, 1-10 J uly 2015

4. T. M isuri, R. A lbertoni, C. Ducci, B. W aldvogel, L. A ppel, R. Eytan, D. Lev, K. Dannenmayer, D. Di Cara, "MEPS: A Low Power Electric Propulsion System for Small Satellites", 10th IAA Symposium on Small Satellites for Earth Observation, Berlin, Germany, 20-23 A pril 2015 